\title{
Assessing Breastfeeding Practices Objectively Using Stable Isotope Techniques
}

\author{
Christine Slater $^{\mathrm{a}}$ Pernille Kaestel $^{\mathrm{b}}$ Lisa Houghton ${ }^{\mathrm{c}}$ \\ ${ }^{\mathrm{a}}$ Independent Consultant, Maryport, UK; ${ }^{\mathrm{b}}$ Division of Human Health, Department of Nuclear Sciences and \\ Applications, International Atomic Energy Agency, Vienna, Austria; ${ }^{C}$ Department of Human Nutrition, University of \\ Otago, Dunedin, New Zealand
}

\section{Keywords}

Breastfeeding practices · Exclusive breastfeeding .

Dose-to-mother technique $\cdot$ Stable isotope $\cdot$ Deuterium

\begin{abstract}
An objective method of assessing breastfeeding practices is required to evaluate progress toward the World Health Organization Global Target 2025: to increase exclusive breastfeeding (EBF) rates in the first 6 months to at least $50 \%$ by 2025. Currently, assessment of EBF at the population level is based on mother or caregiver reporting, which risks recall and social desirability bias. A more objective method is the deuterium oxide dose to mother (DTM) technique, in which lactating mothers are given a small amount of deuteriumlabeled water. The infant receives deuterium during breastfeeding, and a compartmental model is used to determine the amount of human milk consumed by the infant, and the exclusivity of breastfeeding practices. If the amount of human milk consumed by an infant is determined using the DTM technique and the concentration of nutritional components or potentially toxic contaminants is measured, then the infant's intake of essential nutrients or environmental contaminants can be ascertained.
\end{abstract}

(c) International Atomic Energy Agency 2019 Published by S. Karger AG, Basel

\begin{tabular}{ll}
\hline KARGER & $\begin{array}{l}\text { @ International Atomic Energy Agency } 2019 \\
\text { Published by S. Karger AG, Basel }\end{array}$ \\
E-Mail karger@karger.com & $\begin{array}{l}\text { All rights reserved. } \\
\text { This is an Open Access article licensed under the terms of the Creative } \\
\text { www.karger.com/anm } \\
\text { Commons Attribution 3.0 IGO License (CC BY 3.0 IGO)https://cre- } \\
\text { ativecommons.org/licenses/by/3.0/igo/\#). Usage, distribution and re- } \\
\text { production in any medium or format, even for commercial purposes, } \\
\text { is permitted, provided the original work is properly cited. }\end{array}$
\end{tabular}

\section{Introduction}

The World Health Organization recommends exclusive breastfeeding (EBF) of infants for the first 6 months, followed by continued breastfeeding and appropriate complementary feeding up to 2 years of age for optimum health, growth, and development [1]. Although the significance of EBF during early infancy has been specified as a global health priority, EBF rates remain low in many countries. In 2012, the 65th World Health Assembly set a global target to increase EBF rates in the first 6 months after birth from 38\% to at least $50 \%$ by 2025 [2]. An objective, accurate method to assess breastfeeding practices is required to evaluate progress toward this target. Currently, assessment of EBF at the population level is based on mother or caregiver reporting, which risks recall and social desirability bias [3-7]. A more objective method is the deuterium oxide dose to mother (DTM) technique. The DTM method provides an estimate of the amount of human milk consumed by breastfed infants and the exclusivity of breastfeeding practices. Coward et al.

The article is part of the Proceedings of the International Symposium on Understanding the Double Burden of Malnutrition for Effective Interventions organized by the International Atomic Energy Agency (IAEA) in cooperation with United Nations Children's Fund (UNICEF) and World Health Organization (WHO) (10-13 December 2018, Vienna, Austria). 


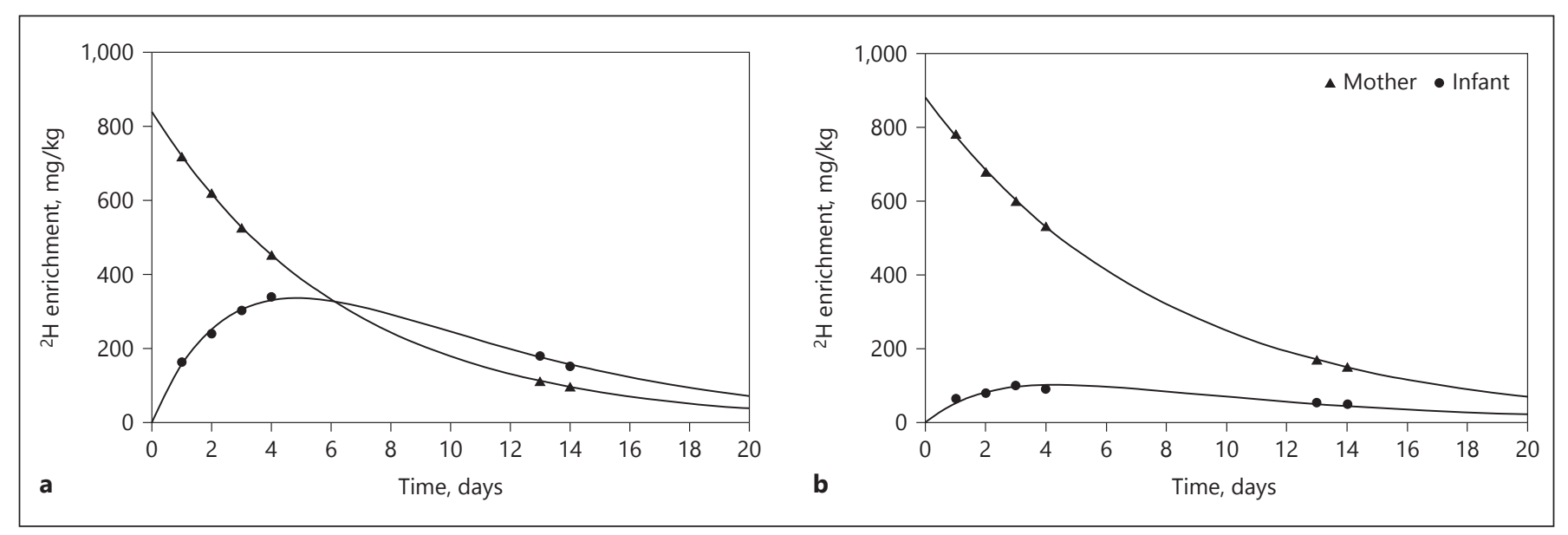

Fig. 1. Deuterium $\left({ }^{2} \mathrm{H}\right)$ enrichment in saliva collected from mothers

) and exclusively (a) or partially (b) breastfed infants $(\bullet)$.

[8] introduced the method in 1982. It uses well-established tracer methods for determining input and output rates in body pools using compartmental modeling techniques. Lactating mothers are given a small amount of deuterium-labeled water. Deuterium is a stable isotope of hydrogen. There is no radiation hazard associated with the use of stable isotope techniques, and there are no adverse effects at the levels consumed in the DTM method [9-11]. The infant receives deuterium during breastfeeding, and a compartmental model is used to determine the outputs: amount of human milk consumed by the infant per day and the exclusivity of breastfeeding practices. The method was subsequently refined during an IAEA Coordinated Research Project, and an Excel spreadsheet was produced to standardize the calculations. This spreadsheet uses the Solver function of Excel to fit the model to the data using the method of least squares. Figure 1 shows examples of the deuterium enrichment in the saliva of mothers and their EBF (a), and partially breastfed (b) infants. The cutoff for EBF was determined empirically as $25 \mathrm{~g}$ water per day from sources other than human milk [12-14]. The method also provides information on the body composition of the mother.

In 2010, da Costa et al. [15] published a pooled analysis ( $n=1,115$ measurements) of the amount of human milk consumed by breastfed infants, aged from 1 week to 24 months. Data were obtained from 737 infants in 12 countries on 5 continents, including Bangladesh, Brazil, Chile, Kenya, Malawi, Mexico, Papua New Guinea, Senegal, The Gambia, USA, UK, and Zambia. The overall human milk intake estimate from the model excluding age effects was 0.778 (95\% CI 0.717-0.839) kg/day. There was a rapid rise from $0.60 \mathrm{~kg} /$ day $(95 \% \mathrm{CI} 0.51-0.70)$ during the first month of life to $0.82 \mathrm{~kg} /$ day $(95 \%$ CI $0.74-0.91)$ at 3-4 months and then very little decline until around 8-9 months. The variability of intake increased in late infancy. Boys consumed $0.05 \mathrm{~kg} /$ day more than girls $(p<0.01)$. Over the past $10-15$ years, through its technical cooperation and coordinated research programs, the IAEA has worked with its Member States in Africa, Asia, and Latin America to establish capacity for using stable isotope techniques to assess breastfeeding practices. An IAEA database has been established containing data on infant feeding and growth (and maternal body composition) from 691 mother/baby pairs from Africa (represented by Benin, Central African Republic, Ghana, Kenya, Morocco, South Africa, Tanzania), 258 pairs from Asia and the Pacific (represented by India, Sri Lanka and Thailand), and 301 pairs from Latin America and the Caribbean (represented by Argentina, Brazil, Chile, Cuba, Dominican Republic, Ecuador, Guatemala, and Uruguay). Although the amount of human milk increases during the first 6 months, when expressed as g or $\mathrm{kg} / \mathrm{day}$, it gradually decreases when expressed as g/day/kg body weight (Fig. 2). Preliminary analysis shows that there is no difference in the amount of human milk consumed per day by exclusively breastfed infants in different regions of the world, when this is expressed relative to the infant's body weight. Two recent IAEA supported studies in Africa in areas with high HIV prevalence (poor, urban areas of Kenya and South Africa) have shown that HIV-infected mothers can exclusively breastfeed their infants without compromising their own body composition $[16,17]$. In Kenya, the HIV-infected women were more likely to exclusively breastfeed their infants than the HIV-uninfected women. South African infants, who were exclusively breastfed for 6 months, had less gut inflammation and higher fat free mass than infants who were not exclusively breastfed $[18,19]$. Studies in poor, ur- 
Fig. 2. Human milk intake over time in g/ day and $\mathrm{g} / \mathrm{kg}$ body weight per day from IAEA database.

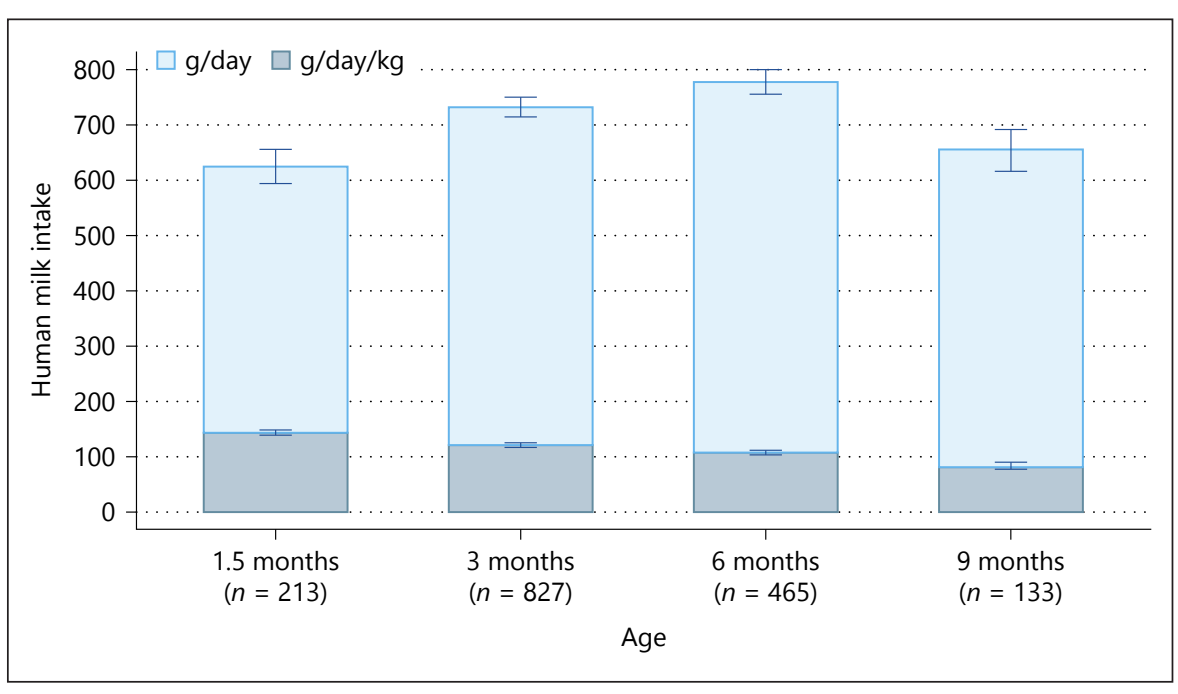

ban areas of Sri Lanka and Bolivia have also used the DTM technique to quantify human milk intake [20-22]. Concerns are sometimes expressed that the lactation performance of undernourished women might be compromised by their nutritional status. A study in rural Pakistan that included women with body mass index ranging from 16.6 to $29.1 \mathrm{~kg} / \mathrm{m}^{2}$ found that EBF tended to be associated with lower maternal body fat, but that there was no evidence for compromised lactational performance due to malnutrition [23]. If the amount of human milk consumed by an infant is measured using the DTM technique and the concentration of nutritional components or potentially toxic contaminants is measured, then the infant's intake of, for example, vitamin A, zinc, arsenic, or other contaminants can be determined [24-30]. Some examples include:

- Studies using this approach have shown that poor vitamin A intake is still a public health concern in Mexico [24]. Infants from urban areas received $66 \%$ of their recommended dietary intake from human milk, but infants from rural areas received only $49 \%$.

- In Senegal, the government was about to introduce fortification of cooking oil with vitamin A with the aim of improving the vitamin A status of women of childbearing age and their children. A study was undertaken to assess 6-months-old infants' vitamin A intake from human milk and vitamin A status of infants and their mothers before the fortification program was widely implemented [25]. The study found that vitamin A deficiency was a concern and the oil fortification program could be a good way to improve the vitamin A status of lactating women and their infants.

- In Bangladesh, it was shown that zinc transferred through breast milk did not differ between predomi- nantly breast-fed infants who were appropriate- and small-for-gestational-age and breast milk zinc concentration was similar to that of wealthier countries [26].

- Infants' exposure to toxic metals (arsenic, lead, and mercury) during breastfeeding has been documented in gold mining areas of Ghana [27], but studies in Bangladesh and Chile have shown that the concentration of toxic elements in human milk is less than in the local drinking water, and therefore lower amounts were transferred to exclusively breastfed infants compared to those who additionally received other foods $[28,29]$.

- Mother to infant transfer of pesticide residues during breastfeeding was measured in agricultural and urban areas of Sonora province, Mexico. The intake did not exceed the acceptable daily intake for humans [30].

The same approach could be used for any nutrient or environmental contaminant.

\section{New Developments}

Although the DTM technique is noninvasive and relatively simple to perform, the need to collect seven saliva samples over a 2 -week period makes it unsuitable for routine use in a public health setting. A less time and resource intensive protocol, capable of distinguishing EBF from non-EBF practices with acceptable accuracy, compared to the full protocol was recently investigated. In doing so, the empirical threshold of $25 \mathrm{~g}$ /day of nonmilk water intake used to distinguish EBF was tested using the DTM technique in a group of healthy Indonesian breastfeeding infants [31]. A total of 121 mother-infant pairs were recruited. The infant feeding practices were observed in the mother's home by trained field assistants recruited from the local community, from 6 am to $6 \mathrm{pm}$ each day on 6 nonconsecu- 
tive days over the 2-week protocol. A pharmacokinetic modeling approach was used to determine the nonmilk water cutoff using a Bayesian framework. The new cutoff is $86.6 \mathrm{~g} /$ day. This value is the maximum water intake that is recommended for an infant to be classified as EBF.

Next, data were pooled from 9 countries including 790 mother-infant pairs to develop and validate a series of shortened sampling designs to assess the intake of water from sources other than human milk using the newly established cutoff value. The full DTM study design was used as the reference (gold standard classification). The new protocol involves 3 sampling times ( 1 pre-dose and 2 post-dose), with a number of post-dose sampling day options that could be selected based on the practicalities of the sampling time frame and an understanding of the level of precision required [32]. The use of the shortened protocol will enable the DTM technique to be used to validate information on breastfeeding practices collected during national nutrition surveys. Furthermore, the ability of the shortened DTM protocol to accurately quantify the volume of human milk consumed by the infant is currently under investigation (unpublished data).

\section{Conclusion}

The DTM technique is a valuable research tool for obtaining objective data on breastfeeding practices and the intake of nutrients and potentially toxic environmental contaminants. A protocol with fewer sampling times, but acceptable accuracy will enable the technique to be used more widely. As such, increased monitoring and evaluation of infant feeding practices will allow countries to better target intervention programs to protect, promote, and support EBF practices of infants up to 6 months of age.

\section{Acknowledgments}

The authors would like to thank all the project coordinators who contributed their data to the IAEA database and the study to develop a shortened protocol for the DTM technique.

\section{Statement of Ethics}

The authors have no ethical conflicts to disclose for this review because there were no humans or animals involved directly.

\section{Disclosure Statement}

C.S. was formerly a Nutrition Specialist at the International Atomic Energy Agency with responsibility for technical cooperation and coordinated research projects using the DTM technique. She retired September 30, 2016. The authors have no other conflicts of interest to declare.

\section{Funding Sources}

The International Atomic Energy Agency made a financial contribution to many of the projects mentioned through the IAEA Technical Cooperation Programme and funding for coordinated research projects.

Open access provided with a grant from the International Atomic Energy Agency.

\section{Author Contributions}

C.S. was the author of the presentation at the DBMal Symposium and prepared the first draft of the manuscript. P.K. was responsible for data cleaning and analysis of DTM data in the IAEA database and preparation of the figures. L.H. prepared the section on "New Developments." All authors critically appraised the manuscript and approved the final version.

\section{Disclaimer}

The statements, opinions and data contained in this publication are solely those of the individual authors and contributors, not of the publishers and the editor(s), and do not necessarily reflect the views of the cooperating organizations, IAEA, UNICEF and WHO. The use of particular designations of countries or territories does not imply any judgement by the cooperating organizations, as to the legal status of such countries or territories, of their authorities and institutions or of the delimitation of their boundaries. The mention of names of specific companies or products (whether or not indicated as registered) does not imply any intention to infringe proprietary rights, nor should it be construed as an endorsement or recommendation on the part of the cooperating organizations.

\section{References}

1 World Health Organization. The optimal duration of exclusive breastfeeding. Geneva, Switzerland: World Health Organization; 2001.

2 World Health Organization. Geneva: Global Targets 2025. To improve maternal, infant and young child nutrition. Geneva: WHO; 2012. https://www.who.int/nutrition/global-target-2025/en/.

3 Moore SE, Prentice AM, Coward WA, Wright A, Frongillo EA, Fulford AJ, et al. Use of stable-isotope techniques to validate infant feeding practices reported by Bangladeshi women receiving breastfeeding counseling. Am J Clin Nutr. 2007 Apr;85(4): 1075-82. 
4 Medoua GN, Sajo Nana EC, Ndzana AC, Makamto CS, Etame LS, Rikong HA, et al. Breastfeeding practices of Cameroonian mothers determined by dietary recall since birth and the dose-to-the-mother deuteriumoxide turnover technique. Matern Child Nutr. 2012 Jul;8(3):330-9.

5 Samuel TM, Thomas T, Bhat S, Kurpad AV. Are infants born in baby-friendly hospitals being exclusively breastfed until 6 months of age? Eur J Clin Nutr. 2012 Apr;66(4):459-65.

6 Mazariegos M, Slater C, Ramirez-Zea M. Validity of Guatemalan mother's self-reported breast-feeding practices of 3-month-old infants. Food Nutr Bull. 2016 Dec;37(4):494-503.

7 Mulol H, Coutsoudis A. Limitations of maternal recall for measuring exclusive breastfeeding rates in South African mothers. Int Breastfeed J. 2018 May;13(1):19.

8 Coward WA, Cole TJ, Sawyer MB, Prentice AM. Breast-milk intake measurement in mixed-fed infants by administration of deuterium oxide to their mothers. Hum Nutr Clin Nutr. 1982;36(2):141-8.

9 Thomson JF. Chapter 7: Effects of D2O in Mammals. Biological Effects of Deuterium. New York: Pergamon Press; 1963.pp. 85-112.

10 Baum D, Dobbing J, Coward WA. Deuterium method for measuring milk intake in babies. Lancet. 1979 Aug;2(8137):309.

11 Jones PJ, Leatherdale ST. Stable isotopes in clinical research: safety reaffirmed. Clin Sci (Lond). 1991 Apr;80(4):277-80.

12 Haisma H, Coward WA, Albernaz E, Visser GH, Wells JC, Wright A, et al. Breast milk and energy intake in exclusively, predominantly, and partially breast-fed infants. Eur J Clin Nutr. 2003 Dec;57(12):1633-42.

13 International Atomic Energy Agency. Stable isotope technique to assess intake of human milk in breastfed infants. IAEA human health series No.7. Vienna: IAEA; 2010. Available from: http://www-pub.iaea.org/MTCD/Publications/PDF/Pub1429_web.pdf.

14 International Atomic Energy Agency. Assessing Intake of Human Milk in Breastfed Infants. IAEA Human Health Campus eLearning. Vienna:IAEA. Available from: https:// humanhealth.iaea.org/HHW/Nutrition/ MilkIntake/index.html.
15 da Costa TH, Haisma H, Wells JC, Mander AP, Whitehead RG, Bluck LJ. How much human milk do infants consume? Data from 12 countries using a standardized stable isotope methodology. J Nutr. 2010 Dec;140(12):2227-32.

16 Mulol H, Coutsoudis A. Breastmilk output in a disadvantaged community with high HIV prevalence as determined by the deuterium oxide dose-to-mother technique. Breastfeed Med. 2016 Mar;11(2):64-9.

17 Oiye S, Mwanda W, Mugambi M, Filteau S, Owino V. Exclusive breastfeeding is more common among HIV-Infected than HIV-uninfected Kenyan mothers at 6 weeks and 6 months postpartum. Breastfeed Med. 2017 Jun;12(5):283-9.

18 Moodley-Govender E, Mulol H, Stauber J, Manary M, Coutsoudis A. Increased exclusivity of breastfeeding associated with reduced gut inflammation in infants. Breastfeed Med. 2015 Dec;10(10):488-92.

19 Mulol H, Coutsoudis A. Association of 6 months of exclusive breastfeeding with higher fat-free mass in infants in a low-resource setting with high HIV prevalence in South Africa. Matern Child Nutr. 2017 Apr;13(2):e12338.

20 Bandara T, Hettiarachchi M, Liyanage C, Amarasena S, Wong WW. The deuterium oxide-to-the-mother method documents adequate breast-milk intake among Sri Lankan infants. J Nutr. 2015 Jun;145(6): 1325-9.

21 Urteaga N, San Miguel JL, Aguilar AM, Muñoz M, Slater C. Nutritional status and human milk intake of exclusively breast-fed infants at high altitude in La Paz, Bolivia. Br J Nutr. 2018 Jul;120(2):158-63.

22 Urteaga N, San Miguel JL, Aguilar AM, Munoz M, Slater C. Nutritional status and human milk intake of exclusively breast-fed infants at high altitude in La Paz, Bolivia - CORRIGENDUM. Br J Nutr. 2018 Nov;120(9):1080.

23 Nazlee N, Bilal R, Latif Z, Bluck L. Maternal body composition and its relationship to infant breast milk intake in rural Pakistan. Food Nutr Sci. 2011 Nov;2(9):932-7.

24 Lopez-Teros V, Limon-Miro AT, AstiazaranGarcia H, Tanumihardjo SA, Tortoledo-Ortiz O, Valencia ME. 'Dose-to-Mother' deuterium oxide dilution technique: an accurate strategy to measure vitamin A intake in breastfed infants. Nutrients. 2017 Feb;9(2): pii:E169.
25 Agne-Djigo A, Idohou-Dossou N, Kwadjode KM, Tanumihardjo SA, Wade S. High prevalence of vitamin A deficiency is detected by the modified relative dose-response test in six-month-old Senegalese breast-fed infants. J Nutr. 2012 Nov;142(11):1991-6.

26 Islam MM, Brown KH. Zinc transferred through breast milk does not differ between appropriate- and small-for-gestational-age, predominantly breast-fed Bangladeshi infants. J Nutr. 2014 May;144(5):771-6.

27 Bansa DK, Awua AK, Boatin R, Adom T, Brown-Appiah EC, Amewosina KK, et al. Cross-sectional assessment of infants' exposure to toxic metals through breast milk in a prospective cohort study of mining communities in Ghana. BMC Public Health. 2017 May;17(1):505.

28 Castro F, Harari F, Llanos M, Vahter M, Ronco AM. Maternal-child transfer of essential and toxic elements through breast milk in a mine-waste polluted area. Am J Perinatol. 2014 Nov;31(11):993-1002.

29 Islam MR, Attia J, Alauddin M, McEvoy M, McElduff P, Slater C, et al. Availability of arsenic in human milk in women and its correlation with arsenic in urine of breastfed children living in arsenic contaminated areas in Bangladesh. Environ Health. 2014 Dec;13(1): 101.

30 Limon-Miro AT, Aldana-Madrid ML, Alvarez-Hernandez G, Antunez-Roman LE, Rodriguez-Olibarria G, Valencia Juillerat ME. Breast milk intake and mother to infant pesticide transfer measured by deuterium oxide dilution in agricultural and urban areas of Mexico. Chemosphere. 2017 Aug;181:682-9.

31 Liu Z, Diana A, Slater C, Preston T, Gibson RS, Houghton L, et al. Development of a nonlinear hierarchical model to describe the disposition of deuterium in mother-infant pairs to assess exclusive breastfeeding practice. J Pharmacokinet Pharmacodyn. 2019 Feb; 46(1):1-13

32 Liu Z, Diana A, Slater C, Preston T, Gibson RS, Houghton L, et al. Development of a parsimonious design for optimal classification of exclusive breastfeeding. CPT Pharmacometrics Syst Pharmacol. 2019 Aug;8(8):596605. 